\title{
Vocalizações de Hyla werneri (Anura, Hylidae) no sul do Brasil
}

\author{
Rodrigo Lingnau', Lorena D. Guimarães e Rogério P. Bastos \\ Departamento de Biologia Geral, Instituto de Ciências Biológicas, Universidade Federal de Goiás, Caixa Postal 131, \\ 74001-970, Goiânia, GO, Brasil. \\ 1 Endereço atual: Rua Tubarão, 321, Bairro América, 89204-340, Joinville, SC, Brasil. E-mail: \\ lingnau@superig.com.br.
}

\begin{abstract}
Vocalizations of Hyla werneri (Anura, Hylidae) in southern Brazil. Herein, we describe the vocal repertoire of Hyla werneri. We found four distinct vocalizations: advertisement call, aggressive call, distress call, and a mixed call. The advertisement call was the most frequent vocalization, with one to 23 notes. Aggressive calls had only one note, but with longer duration than notes of advertisement calls. One individual emitted four distress calls, and these showed a great variation. The mixed calls are formed by the emission of an aggressive call followed by five to 23 notes similar to the advertisement call, probably a strategy to partition the attractive and aggressive functions in different notes on the same call. We suggest that the mixed calls of $H$. werneri could be a graded aggressive call system, already found in other anurans, which allows males to gradually reduce the number of attractive elements while increasing the aggressiveness of the call.
\end{abstract}

Keywords: Anura, Hylidae, Hyla werneri, Hyla microcephala group, vocalization, complex calls.

Palavras-chave: Anura, Hylidae, Hyla werneri, Hyla grupo microcephala, vocalização, cantos complexos.

\section{Introdução}

A produção de sons por animais, particularmente os anfíbios anuros, tem como função primária anunciar a presença de um indivíduo a outros da mesma espécie (Duellman e Trueb 1994). Algumas espécies apresentam amplo repertório vocal, empregando cantos distintos dependendo do contexto social (Wells 1977, Guimarães e Bastos 2003, Wogel et al. 2004).

Recebido em 10 de junho de 2004.

Aceito em 12 de novembro de 2004

Distribuído em dezembro de 2004.
As características das vocalizações, tais como duração do canto, taxa de repetição e freqüência, podem sofrer influência de diversos fatores abióticos. Em certas espécies, um aumento da temperatura acarreta um aumento da taxa de repetição e/ou da duração do canto (Sullivan 1992, Giacoma et al. 1997), enquanto a freqüência do canto está geralmente correlacionada com o tamanho do macho (Márquez e Bosch 2001, Bueno et al. 2003). Devido à importância da vocalização de anúncio como mecanismo de isolamento reprodutivo prézigótico, parâmetros do canto têm sido utilizados em estudos taxonômicos e sistemáticos para 
confirmar a identidade de algumas espécies ou distinguir espécies morfologicamente similares (De la Riva et al. 1996, Garcia et al. 2001).

Neste trabalho, foram estudadas as vocalizações de Hyla werneri Cochran, 1952. Essa espécie ocorre do sul do Estado de São Paulo até Santa Catarina e pertence ao grupo de Hyla microcephala Cope, 1886 (Pombal e Bastos 1998). Nossos objetivos foram apresentar o repertório vocal de $H$. werneri e verificar se existe correlação dos parâmetros acústicos do canto de anúncio com a temperatura do ar ou com o comprimento rostro-cloacal (CRC) dos machos.

\section{Material e Métodos}

As observações foram realizadas na Estação II do Instituto Agronômico do Paraná (2526’59”' S, 4852'09'” W), Morretes, Estado do Paraná, entre janeiro e abril de 2002. O local de estudo compreende várias poças temporárias ao redor de uma área com cultivo de arroz. Os turnos de observação eram iniciados logo após o pôr-do-sol e estendiam-se até aproximadamente meia-noite. O trabalho envolveu um total de 26 horas de observação, em cinco visitas à área de estudo. Os anuros foram observados e coletados com auxílio de lanterna, segundo os métodos de animal focal e todas as ocorrências (Martin e Bateson 1986). Após a localização dos machos por meio de suas vocalizações, os cantos foram registrados com o auxílio de um microfone Sony ECM acoplado a um gravador DAT TCD-D100, a cerca de $30 \mathrm{~cm}$ do macho emissor. Posteriormente, as vocalizações foram editadas com freqüência de $22 \mathrm{kHz}$ e resolução de 16 bits em computador PC Pentium e analisadas com os programas Avisoft Sonagraph Light e Cool Edit 96. Informações acerca da freqüência dominante foram obtidas pela Transformação Rápida de Fourier (FFT, 1024 pontos). Não foi possível obter o mesmo número de gravações de todos os tipos de vocalizações, e, quando possível, foram selecionados aleatoriamente cinco cantos de cada tipo de cada macho gravado. A termino- logia utilizada na descrição dos cantos seguiu Duellman e Trueb (1994) e Gerhardt (1998). A temperatura do ar foi medida com termômetro digital posicionado sobre o solo próximo ao sítio de vocalização dos machos. Os indivíduos gravados foram coletados e seu CRC foi medido com paquímetro digital (precisão de 0,01 mm). As gravações foram arquivadas na Coleção de Arquivos Sonoros do Laboratório de Comportamento Animal do Departamento de Biologia Geral da Universidade Federal de Goiás, e os indivíduos que tiveram seus cantos gravados foram depositados na coleção de anfíbios do Museu Nacional, Rio de Janeiro (MNRJ 3038830402). As características acústicas analisadas foram a duração do canto, o número de notas por canto, a duração das notas, o número de pulsos por nota e a freqüência dominante. Cada medida é apresentada como média \pm desvio padrão ( $\bar{X} \pm \mathrm{DP})$. Para as correlações das características acústicas com a temperatura do ar ou com o CRC, foi utilizado o coeficiente de correlação de Pearson (Zar 1996) com nível de significância de 0,05.

\section{Resultados}

Machos de $H$. werneri iniciaram suas vocalizações logo após o pôr-do-sol, estendendo-se até após meia-noite, e foram observados sobre ramos da vegetação próximos ao solo, até a uma altura de $50 \mathrm{~cm}$. O repertório vocal dos machos consistiu de quatro vocalizações com características distintas: canto de anúncio, canto agressivo, canto de agonia e canto misto (Tabela 1).

A vocalização mais freqüentemente emitida pelos machos foi considerada como canto de anúncio (Figura 1). Os principais parâmetros acústicos desse canto estão na Tabela 1. A freqüência fundamental do canto de anúncio oscilou entre 2,9 e 3,4 kHz. Os cantos agressivos (Figura 2) são formados por uma única nota pulsionada e seus principais parâmetros acústicos podem ser encontrados na Tabela 1 . Os machos emitiam cantos agressivos em res- 
Tabela 1 - Valores médios seguidos do desvio padrão e amplitude (entre parênteses) dos parâmetros das vocalizações de Hyla werneri em Morretes, Estado do Paraná, sul do Brasil.

\begin{tabular}{|c|c|c|c|c|c|}
\hline & \multirow{2}{*}{$\begin{array}{l}\text { CANTO DE } \\
\text { ANÚNCIO }\end{array}$} & \multirow{2}{*}{$\begin{array}{c}\text { CANTO } \\
\text { AGRESSIVO }\end{array}$} & \multirow{2}{*}{$\begin{array}{l}\text { CANTO DE } \\
\text { AGONIA }\end{array}$} & \multicolumn{2}{|c|}{ CANTO MISTO } \\
\hline & & & & 1a Parte & 2a Parte \\
\hline Duração do canto (s) & $\begin{array}{c}0,517 \pm 0,3 \\
(0,02-1,77)\end{array}$ & $\begin{array}{c}0,147 \pm 0,008 \\
(0,092-0,229)\end{array}$ & $\begin{array}{c}0,037 \pm 0,021 \\
(0,027-0,061)\end{array}$ & $\begin{array}{c}0,145 \pm 0,025 \\
(0,116-0,203)\end{array}$ & $\begin{array}{c}1,275 \pm 0,458 \\
(0,8-2,516)\end{array}$ \\
\hline Número de notas & $\begin{array}{l}4,06 \pm 1,98 \\
\quad(1-12)\end{array}$ & 1 & 1 & 1 & $\begin{array}{c}11,714 \pm 3,58 \\
(5-23)\end{array}$ \\
\hline Duração das notas (s) & $\begin{array}{c}0,023 \pm 0,004 \\
(0,013-0,040)\end{array}$ & $\begin{array}{c}0,147 \pm 0,008 \\
(0,092-0,229)\end{array}$ & $\begin{array}{c}0,037 \pm 0,021 \\
(0,027-0,061)\end{array}$ & $\begin{array}{c}0,145 \pm 0,025 \\
(0,116-0,203)\end{array}$ & $\begin{array}{l}0,014 \pm 0,002 \\
(0,01-0,018)\end{array}$ \\
\hline $\begin{array}{l}\text { Número de pulsos por } \\
\text { nota }\end{array}$ & $\begin{array}{l}6,14 \pm 1,09 \\
\quad(3-10)\end{array}$ & $\begin{array}{c}36,27 \pm 7,3 \\
(24-54)\end{array}$ & $\begin{array}{l}4,5 \pm 3,69 \\
(1-9)\end{array}$ & $\begin{array}{c}28,14 \pm 6,75 \\
(21-45)\end{array}$ & $\begin{array}{l}3,07 \pm 0,61 \\
\quad(2-4)\end{array}$ \\
\hline $\begin{array}{l}\text { Frequiência dominante } \\
(\mathrm{kHz})\end{array}$ & $\begin{array}{l}6,75 \pm 0,28 \\
(6,33-7,41)\end{array}$ & $\begin{array}{c}6,88 \pm 0,33 \\
(6,32-7,59)\end{array}$ & $\begin{array}{c}5,77 \pm 0,45 \\
(5,39-6,43)\end{array}$ & $\begin{array}{c}6,47 \pm 0,11 \\
(6,25-6,68)\end{array}$ & $\begin{array}{c}6,72 \pm 0,15 \\
(6,36-6,75)\end{array}$ \\
\hline $\begin{array}{l}\mathrm{N} \text { (cantos analisados / } \\
\sigma^{\pi} \text { gravados) }\end{array}$ & $75 / 15$ & $15 / 3$ & $4 / 1$ & $14 / 3$ & \\
\hline
\end{tabular}

posta a machos vizinhos que estivessem emitindo cantos de anúncio ou mesmo cantos agressivos. Os machos também podiam emitir uma seqüência de 5 a 23 notas similares ao canto de anúncio logo após uma nota similar ao canto agressivo, que chamamos de canto misto (Figura 3). Esse canto é formado por duas partes distintas, sendo que a primeira parte é formada por uma única nota, muito similar ao canto agressivo, e a segunda parte é formada por 5 a 23 notas muito similares às notas do canto de anúncio. Os principais parâmetros acústicos das duas partes do canto misto podem ser encontrados na Tabela 1. Os três machos que emitiram esses cantos mistos encontravam-se em coros densos, e geralmente os vizinhos mais próximos respondiam também com o canto misto, formando coros de cantos mistos. Um macho emitiu cantos de agonia (Figura 4) ao ser manipulado. O indivíduo havia sido coletado e mantido durante a noite em um saco plástico umedecido com água da poça onde foi encontrado. Na manhã seguinte, antes de proceder-se à sua fixação, enquanto era manipulado, começou a emitir cantos de agonia. Antes de iniciar-se a gravação de sua vocalização, o macho já havia emitido cinco cantos de agonia, os quais soaram bastante diferentes ao ouvido, e somente mais quatro cantos puderam ser gravados, enquanto o indivíduo continuava a ser manipulado. Os quatro cantos gravados são bem variáveis, e seus principais parâmetros acústicos constam da Tabela 1.

O tamanho médio dos machos gravados e coletados foi de $17 \pm 1,27 \mathrm{~mm}(15,14$ a 19,98 $\mathrm{mm}$ ) e a temperatura do ar na ocasião da gravação variou de 19,7 a $25,3^{\circ} \mathrm{C}$. Não foram encontradas correlações significativas entre a temperatura do ar e quaisquer dos parâmetros temporais do canto de anúncio: duração do canto ( $\mathrm{r}=-0,1 ; \mathrm{N}=15 ; \mathrm{P}>0,05)$, número de notas $(r=0,1 ; N=15 ; P>0,05)$, duração das notas $(\mathrm{r}=-0,1 ; \mathrm{N}=15 ; \mathrm{P}>0,05)$ e número de 

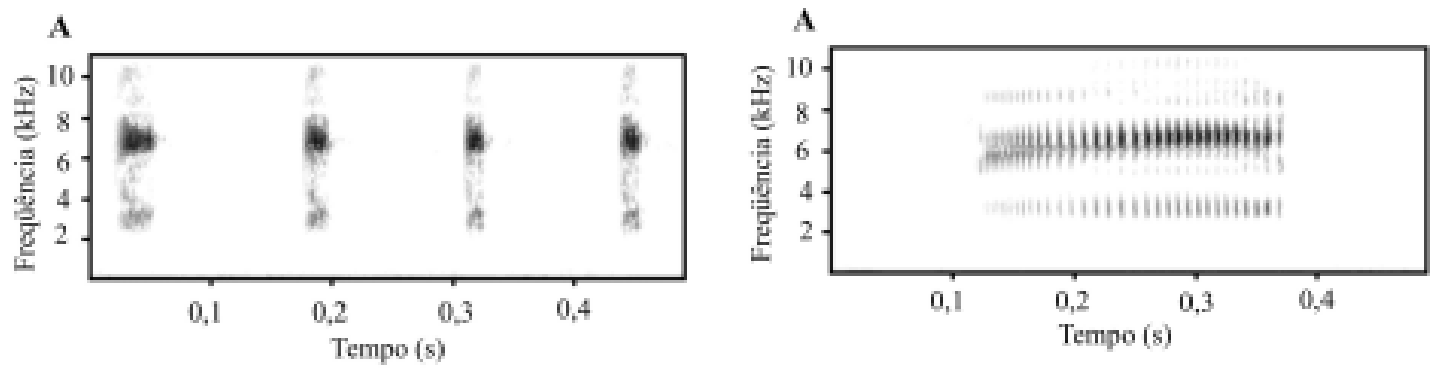

B

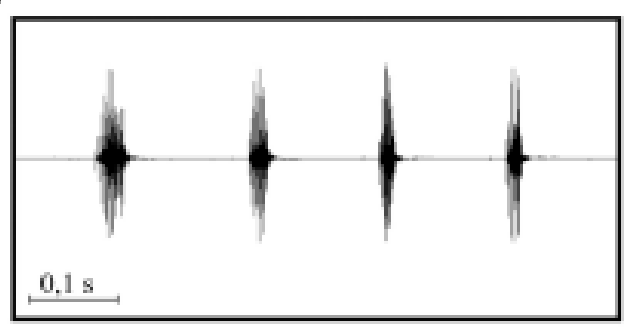

B

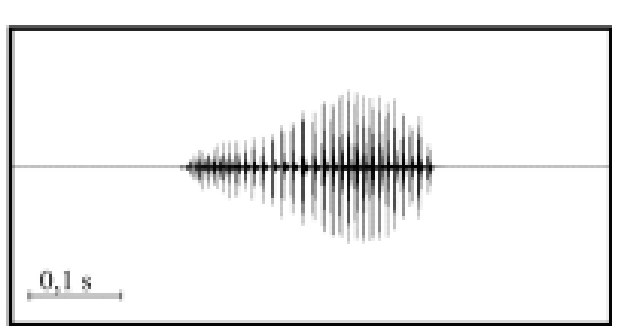

Figura 1 - Sonograma (A) e oscilograma (B) do canto de anúncio de Hyla werneri em Morretes, Estado do Paraná, sul do Brasil (MNRJ 30390, temperatura do ar $\left.25,3^{\circ} \mathrm{C}\right)$.

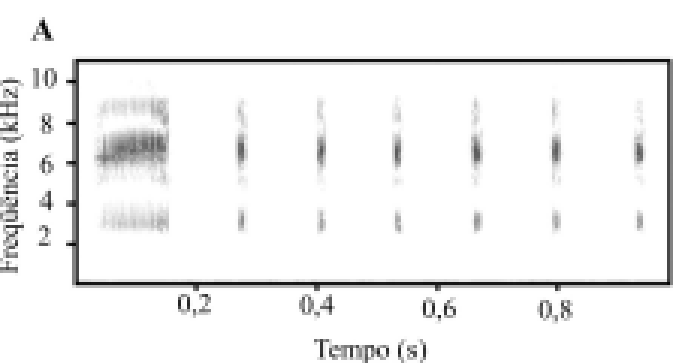

B

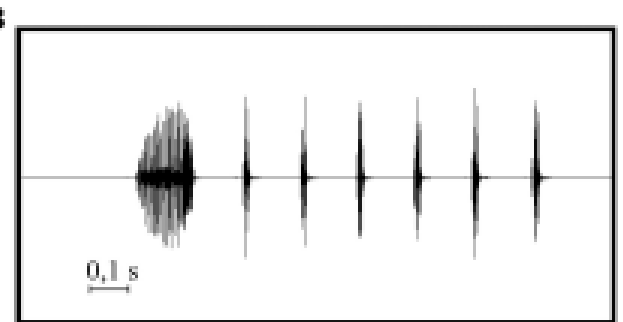

Figura 3 - Sonograma (A) e oscilograma (B) do canto misto de Hyla werneri em Morretes, Estado do Paraná, sul do Brasil (MNRJ 30392, temperatura do ar $22,2^{\circ} \mathrm{C}$ ).

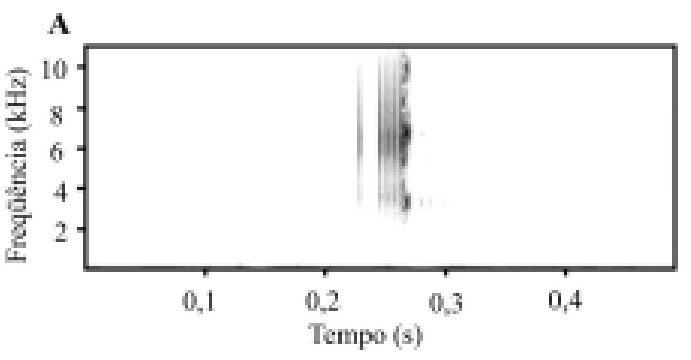

Figura 2 - Sonograma (A) e oscilograma (B) do canto agressivo de Hyla werneri em Morretes, Estado do Paraná, sul do Brasil (MNRJ 30392, temperatura do ar $22,2^{\circ} \mathrm{C}$ ).

B

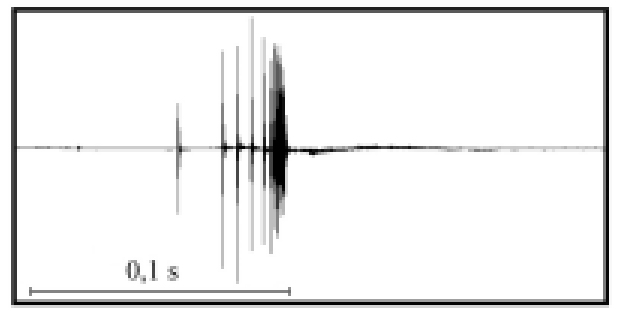

Figura 4 - Sonograma (A) e oscilograma (B) do canto de agonia de Hyla werneri em Morretes, Estado do Paraná, sul do Brasil (MNRJ 30390, temperatura do ar $25,3^{\circ} \mathrm{C}$ ). 
pulsos por nota $(\mathrm{r}=0,01 ; \mathrm{N}=15 ; \mathrm{P}>0,05)$. Também não foi encontrada correlação significativa entre a freqüência dominante do canto de anúncio e o CRC ( $\mathrm{r}=-0,45 ; \mathrm{N}=15 ; \mathrm{P}>0,05)$.

\section{Discussão}

Em algumas espécies de anuros, foram encontradas correlações significativas entre parâmetros acústicos e a temperatura do ar ou o tamanho dos machos (Giacoma et al. 1997, Navas e Bevier 2001, Bueno et al. 2003). Em Hyla werneri, não foi detectada nenhuma influência da temperatura sobre quaisquer dos parâmetros acústicos. Há uma correlação significativa entre a freqüência dominante e o CRC em H. cruzi (Bueno et al. 2003), pertencente ao mesmo grupo que $H$. werneri. O fato dos parâmetros acústicos não estarem correlacionados significativamente com a temperatura do ar e com o CRC dos machos de $H$. werneri pode ser uma indicação de que outros fatores ecológicos (e.g., densidade do coro) podem ter uma maior influência sobre a atividade de vocalização dessa espécie, como relatado para outros anuros (Boatright-Horowitz et al. 2000). Outro fato que pode justificar a ausência de correlações significativas é a pequena variação da temperatura do ar, não fornecendo, dessa forma, evidências suficientes para demonstrar alguma influência desse fator na vocalização.

São poucas as espécies pertencentes ao grupo de $H$. microcephala que já têm o seu canto de anúncio descrito, principalmente aquelas ocorrentes ao longo da Mata Atlântica. Lingnau e Bastos (2003) relataram que o canto de anúncio de $H$. werneri é diferente do canto de duas espécies aparentadas que já tiveram seus cantos de anúncio descritos, $H$. decipiens e $H$. meridiana, o que reforça sua distinção específica.

Neste trabalho, foi documentada a ocorrência de cantos mistos em Hyla werneri, em que os machos aparentemente combinam o canto agressivo com o canto de anúncio. Comparando as Figuras 1 e 2 com a Figura 3, é possível visualizar como o canto misto parece ser formado pela junção desses cantos. Wells (1988) sugeriu que cantos mistos codificam as funções de atração e agressividade em notas diferentes, sendo assim uma estratégia para compartilhar as duas funções e reduzir os gastos energéticos das vocalizações agressivas, já que sinais agressivos não são atraentes às fêmeas. Não descartamos também a possibilidade de que os cantos mistos encontrados em $H$. werneri sejam também um sistema gradativo de comunicação (graded aggressive calls, sensu Wells 1988). Esse sistema permite a um macho reduzir gradativamente o número de elementos atraentes enquanto aumenta a agressividade do canto à medida que outro macho se aproxima. Isso representa um canto misto mais refinado, que já foi documentado para outras espécies do grupo de $H$. microcephala, como $H$. phlebodes (Schwartz e Wells 1984) e H. microcephala (Schwartz e Wells 1985).

Anuros agarrados por um predador podem emitir cantos aos quais se aplica o nome de canto de agonia (Duellman e Trueb 1994). Sugeriu-se que esses cantos servem para assustar o predador que esteja manipulando o animal ou atrair predadores secundários capazes de interferir na predação (Hödl e Gollmann 1986). Para o único indivíduo de Hyla werneri para o qual foram obtidos esses cantos, a freqüência dominante destes foi menor que a dos cantos de anúncio e agressivo. Houve uma considerável variação entre os quatro cantos de agonia do único indivíduo gravado (Tabela 1). Segundo Martins e Haddad (1988), variações intraespecíficas e mesmo individuais de cantos de agonia podem estar relacionadas ao grau de agonia sofrido pelo animal, fato que explica também a variação encontrada nesses cantos em $H$. werneri.

\section{Agradecimentos}

À diretoria do Instituto Agronômico do Paraná, principalmente à diretoria local da Estação Experimental de Morretes, pela 
permissão de acesso ao local de estudo e por todas as facilidades oferecidas. Por auxílio nos trabalhos de campo, agradecemos a Rafael Costa e a Carina M. Lingnau. Aos dois revisores anônimos por sugestões apresentadas. RL e LDG agradecem à CAPES pela bolsa concedida, e RPB agradece ao CNPq pela Bolsa de Produtividade em Pesquisa. Este trabalho foi efetuado sob Licença de Coleta IBAMA 155/01.

\section{Referências Bibliográficas}

Boatright-Horowitz, S. L., S. S. Horowitz e A. M. Simmons. 2000. Patterns of vocal interactions in a bullfrog (Rana catesbeiana) chorus: preferential responding to far neighbors. Ethology 106: 701-712.

Bueno, M. A. F., S. L. Dutra, L. P. Lima e R. P. Bastos. 2003. Padrão de vocalização de anúncio em espécies de Hylidae (Anura) do Brasil Central. Comunicações do Museu de Ciências e Tecnologia da PUCRS, Série Zoologia 16: 39-51.

De la Riva, I., J. Bosch e R. Márquez. 1996. Advertisement calls of two Bolivian toads (Anura: Bufonidae: Bufo). Herpetological Journal 6: 59-61.

Duellman, W. E. e L. Trueb. 1994. Biology of Amphibians. $2^{\mathrm{a}}$ ed. Baltimore e London. The John Hopkins University Press. 670 pp.

Garcia, P. C. A., U. Caramaschi e A. Kwet. 2001. O status taxonômico de Hyla cochranae Mertens e recaracterização de Aplastodiscus A. Lutz (Anura, Hylidae). Revista Brasileira de Zoologia 18: 1197-1218.

Gerhardt, H. C. 1998. Acoustic signals of animals: recordings, field measurements, analysis and description. Pp. 1-25 in S. L. Hopp, M. J. Owren e C. S. Evans (eds.), Animal Acoustic Communication. Berlin. Springer Verlag.

Giacoma, C., C. Zugolaro e L. Beani. 1997. The advertisement calls of the green toad (Bufo viridis): variability and role in mate choice. Herpetologica 53: 454-464.

Guimarães, L. D. e R. P. Bastos. 2003. Vocalizações e interações acústicas em Hyla raniceps (Anura, Hylidae) durante a atividade reprodutiva. Iheringia, Série Zoologia 93: 149-158.

Hödl, W. e G. Gollmann. 1986. Distress calls in neotropical frogs. Amphibia-Reptilia 7: 11-21.

Lingnau, R. e R. P. Bastos. 2003. Vocalizações de duas espécies de anuros do sul do Brasil (Amphibia, Hylidae). Arquivos do Museu Nacional 61: 203-207.

Márquez, R. e J. Bosch. 2001. Communication and mating in the midwife toads (Alytes obstetricans and Alytes cisternasii). Pp. 220-231 in M. J. Ryan (ed.), Anuran Communication. Washington. Smithsonian Institution Press.

Martin, P. e P. Bateson. 1986. Measuring Behavior - an introductory guide. Cambridge. Cambrige University Press. 199 pp.

Martins, M. e C. F. B. Haddad. 1988. Vocalizations and reproductive behaviour in the smith frog, Hyla faber Wied (Amphibia: Hylidae). Amphibia-Reptilia 9: 4960.

Navas, C. A. e C. R. Bevier. 2001. Thermal dependency of calling performance in the eurythermic frog Colostethus subpunctatus. Herpetologica 57: 384-395.

Pombal, J. P., Jr. e R. P. Bastos. 1998. Nova espécie de Hyla Laurenti, 1768 do centro-oeste brasileiro e a posição taxonômica de Hyla microcephala werneri Cochran, 1952 e Hyla microcephala meridiana B. Lutz, 1952 (Anura, Hylidae). Boletim do Museu Nacional 390: 1-14.

Schwartz, J. J. e K. D. Wells. 1984. Vocal behavior of the neotropical treefrog Hyla phlebodes. Herpetologica 40: 452-463.

Schwartz, J. J. e K. D. Wells. 1985. Intra- and interspecific vocal behavior of the neotropical treefrog Hyla microcephala. Copeia 1985: 27-38.

Sullivan, B. K. 1992. Calling behavior of the southwestern toad (Bufo microscaphus). Herpetologica 48: 383389.

Wells, K. D. 1977. The social behaviour of anuran amphibians. Animal Behaviour 25: 666-693.

Wells, K. D. 1988. The effect of social interactions on anuran vocal behavior. Pp. 433-454. in B. Fritzsch, M. J. Ryan, W. Wilczynski, T. E. Hetherington e W. Walkowiak (eds.), The Evolution of the Amphibian Auditory System. New York. John Wiley and Sons.

Wogel, H., P. A. Abrunhosa e J. P. Pombal Jr. 2004. Vocalizations and aggressive behavior of Phyllomedusa rohdei (Anura: Hylidae). Herpetological Review 35: 239-243.

Zar, J. H. 1996. Biostatistical Analysis. $3^{\mathrm{a}}$ ed. New Jersey. Prentice-Hall Press. 662 pp. 